University of New Hampshire

University of New Hampshire Scholars' Repository

Physics Scholarship

Physics

$4-1-2004$

\title{
Energetic particle sounding of the magnetopause: A contribution by Cluster/RAPID
}

Q. Zong

T. A. Fritz

Harlan E. Spence

Boston University, harlan.spence@unh.edu

K. Oksavik

Z. Y. Pu

See next page for additional authors

Follow this and additional works at: https://scholars.unh.edu/physics_facpub

Part of the Physics Commons

\section{Recommended Citation}

Zong, Q.-G., T. A. Fritz, H. Spence, K. Oksavik, Z.-Y. Pu, A. Korth, and P. W. Daly (2004), Energetic particle sounding of the magnetopause: A contribution by Cluster/RAPID, J. Geophys. Res., 109, A04207, doi:10.1029/2003JA009929.

This Article is brought to you for free and open access by the Physics at University of New Hampshire Scholars' Repository. It has been accepted for inclusion in Physics Scholarship by an authorized administrator of University of New Hampshire Scholars' Repository. For more information, please contact Scholarly.Communication@unh.edu. 
Authors

Q. Zong, T. A. Fritz, Harlan E. Spence, K. Oksavik, Z. Y. Pu, A. Korth, and P. W. Daly 


\title{
Energetic particle sounding of the magnetopause: A contribution by Cluster/RAPID
}

\author{
Q.-G. Zong, ${ }^{1}$ T. A. Fritz, ${ }^{1}$ H. Spence, ${ }^{1}$ K. Oksavik, ${ }^{2,3}$ Z.-Y. Pu, ${ }^{4}$ A. Korth, ${ }^{5}$ and P. W. Daly ${ }^{5}$
}

Received 10 March 2003; revised 24 January 2004; accepted 18 February 2004; published 7 April 2004.

[1] In this paper we present new results using Cluster/Research with Adaptive Particle Imaging Detectors (RAPID) energetic particle observations to remotely sound the highlatitude magnetopause in three dimensions. We demonstrate that energetic particle flux variations in the vicinity of the magnetopause (inside the magnetosphere) are mainly modulated by the absorbing magnetopause during quiet geomagnetic conditions. Less than two gyro radii from an absorbing boundary a trapped particle distribution becomes nongyrotropic, as particles start to encounter the boundary. Knowing the magnetic field and the particle mass and energy, the direction and distance to the magnetopause can be derived by examining the azimuthal distribution of locally mirroring particles. Combining observations from three nearby spacecraft gives a three-dimensional, local picture of the magnetopause surface. We exploit anisotropic ion distributions to determine magnetopause distances, orientations, and structures in the interval from 1320 to 1420 UT on 14 January 2001 for the three Cluster spacecraft (Rumba, Samba, and Tango) located on the duskside (at $\sim 1700$ MLT) high-latitude region. The results clearly illustrate that the magnetopause ion sounding technique as proposed by Williams [1979], Fritz and Fahnenstiel [1982], and Zong et al. [2000] can be used to remotely study the three-dimensional orientation and location of the magnetopause surface and the gradient variation of the plasma parameters. Intercomparison between energetic particle sounding distance and simultaneous plasma and magnetic field measurements suggests that solar wind plasma can penetrate more than $\approx 1000 \mathrm{~km}$ deeper than the trapping boundary. The fluxes of different ion species are proportional to the distance from the magnetopause, with a correlation coefficient of 0.7 to 0.8 . The energetic proton flux gradient as a function of distance from the magnetosphere is about 100 particles $\mathrm{cm}^{-2} \mathrm{~s}^{-1} \mathrm{sr}^{-1}$ per kilometer. In contrast, the solar wind plasma density is found to be inversely proportional to the distance. The solar wind plasma appears to be bounded by an exponential with an e-folding distance of $1000 \mathrm{~km}$. INDEX TERMS: 2724 Magnetospheric Physics: Magnetopause, cusp, and boundary layers; 2720 Magnetospheric Physics: Energetic particles, trapped; 2728 Magnetospheric Physics: Magnetosheath; 2740 Magnetospheric Physics:

Magnetospheric configuration and dynamics; KEYWORDS: energetic particles, ion sounding, magnetopause

Citation: Zong, Q.-G., T. A. Fritz, H. Spence, K. Oksavik, Z.-Y. Pu, A. Korth, and P. W. Daly (2004), Energetic particle sounding of the magnetopause: A contribution by Cluster/RAPID, J. Geophys. Res., 109, A04207, doi:10.1029/2003JA009929.

\section{Introduction}

[2] Energetic ions are ideally suited to sound the magnetospheric boundaries at large distances (up to two gyroradii, $\rho$ ) from the satellite. This was first suggested and successfully used near the magnetopause by Konradi and Kaufmann [1965] and Kaufmann and Konradi [1973]. This concept is shown schematically in Figure 1. The azimuthal distribution of particle fluxes exhibits a strong anisotropy indicating that the satellite is close (within two ion gyro-

\footnotetext{
USA.

${ }^{1}$ Center for Space Physics, Boston University, Boston, Massachusetts,

${ }^{2}$ Department of Physics, University of Bergen, Bergen, Norway.

${ }^{3}$ Now at Department of Physics, University of Oslo, Oslo, Norway.

${ }^{4}$ Department of Geophysics, Peking University, Beijing, China.

${ }^{5}$ Max-Planck-Institut für Aeronomie, Katlenburg-Lindau, Germany.
}

Copyright 2004 by the American Geophysical Union. 0148-0227/04/2003JA009929 radii) of an absorbing boundary associated with the magnetopause. With the satellite located at the origin, it is seen that particles with arrival directions counter-clockwise from $\phi_{A}$ to $\phi_{D}$ can complete their gyro orbits while those from $\phi_{D}$ to $\phi_{A}$ will contact the magnetopause and be scattered or lost, thus reducing the flux from this direction. From these angles a unique perpendicular distance $R$ and orientation angle $\beta$ to the magnetopause plane can be calculated. The distance and the orientation angle of the magnetopause $\beta$ are given by [Zong et al., 2000]

$$
\begin{aligned}
& \beta=90^{\circ}-\frac{\phi_{A}+\phi_{D}}{2}, \\
& R=\rho\left[1-\sin \left(90^{\circ}+\frac{\phi_{D}-\phi_{A}}{2}\right)\right],
\end{aligned}
$$

where $\rho$ is the gyro-radius of the sounding ions, $R$ is the distance to the magnetopause which is less than $2 \rho$. We 

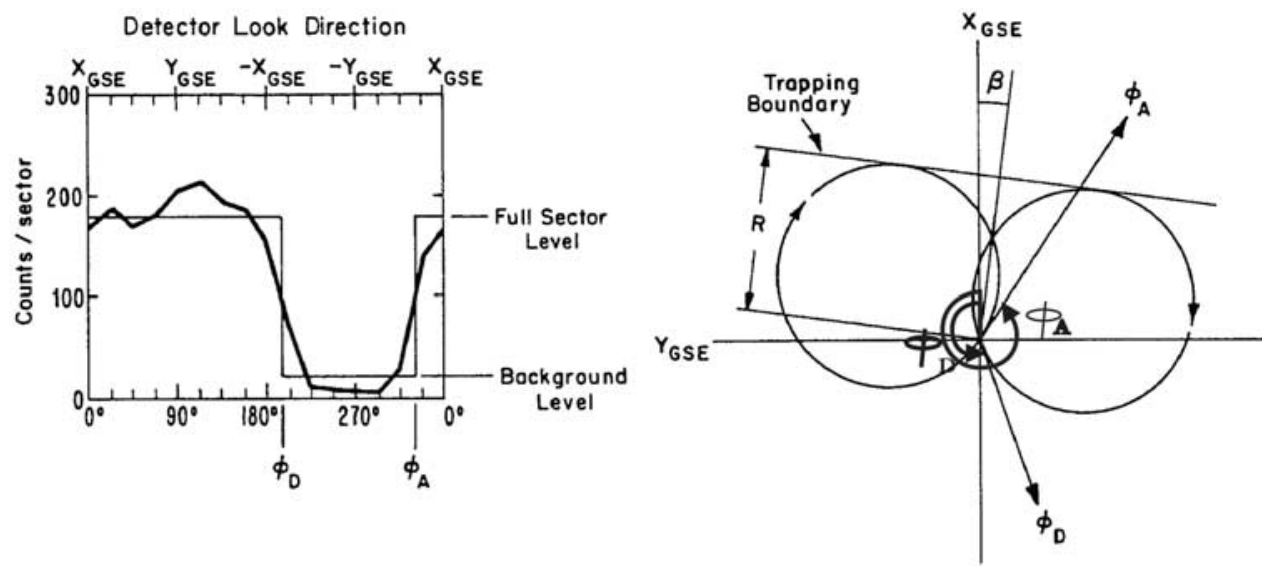

Figure 1. Technique for determining trapping boundary parameter near the dayside magnetopause. At the left of this figure is the 16 sector normalized partially trapped locally mirroring ion $(>24 \mathrm{keV})$ distribution. Superimposed is a square wave fit from which the angles $\phi_{D}$ and $\phi_{A}$ are obtained. The drop in count rate from $\phi_{D}$ to $\phi_{A}$ is interpreted as scattering from a trapping boundary as shown in the portion of the figure on the right. From these angles, the perpendicular distance $R$ and orientation angle $\beta$ to the trapping boundary are obtained [after Fritz and Fahnenstiel, 1982].

assume that the flux is uniform when the spacecraft is far enough away from the magnetopause (i.e., the distance $\mathrm{R}$ is larger than $2 \rho$ ). If the angular resolution of an instrument is $\delta \phi$, and if the uncertainty in gyro-radius caused by the width of the energy channel used for sounding is $\delta \rho$, then the errors of distance $R$ and the orientation angle of the magnetopause $\beta$ can be written as

$$
\begin{aligned}
\Delta \beta & = \pm \Delta \phi \\
\Delta R & = \pm\left[\sqrt{\left(2 R \rho-R^{2}\right)} \Delta \phi+\left(\frac{R}{\rho}\right) \Delta \rho\right]
\end{aligned}
$$

[3] Now, this technique has been applied widely to sense boundaries and boundary motions such as: (1) to probe the location, thickness, orientation, and velocity of the magnetopause [Konradi and Kaufmann, 1965; Kaufmann and Konradi, 1973; Williams, 1979; Zong et al., 2000]; (2) boundary waves on the magnetopause [Williams, 1980; Fritz and Fahnenstiel, 1982; Zong et al., 2000]; (3) the last closed field line of the dayside magnetosphere [e.g., Fritz and Fahnenstiel, 1982; Daly, 1982]; (4) the boundary of flux transfer events (FTE) [Daly, 1982]; and (5) the plasma sheet and plasma sheet boundary layer [Daly et al., 1984]. More recently, this method was also applied to sound the high-latitude magnetospheric boundary using multispacecraft Cluster/RAPID data [Oksavik et al., 2002].

[4] The 14 January 2001 event studied in our paper has been investigated widely [Moen et al., 2001; Opgenoorth et al., 2001; Lockwood et al., 2001; Zong et al., 2002]. An overview of this event has been given by Opgenoorth et al. [2001], who focus on transient flow signatures in the postnoon ionosphere during the passage of Cluster through the interior particle cusp. Lockwood et al. [2001] emphasize the link between Cluster observations and transient signatures seen with the EISCAT Svalbard Radar, and Moen et al. [2001] present optical observations of the dayside aurora over Svalbard. During this event, the IMF was essential northward, and geomagnetic activity was very quiet. How- ever, abundant energetic ions were observed in the highlatitude boundary region, in a layer located just inside the magnetosphere. The energetic particles in this region are highly anisotropic and their fluxes vary. The reason for the anisotropic particle distributions and the modulation mechanism of energetic particle flux has not yet been discussed [Zong et al., 2002]. By exploiting the anisotropic ion distribution, in this paper, we obtain information about the surface of the magnetopause in three dimensions, the location of the magnetopause, and the distance to the magnetopause. The relation between the plasma parameters and the distance to magnetopause is discussed. It is further demonstrated that the energetic particle flux variations are mainly modulated by the distance to the absorbing magnetopause during geomagnetically quiet conditions.

\section{RAPID Instrument}

[5] The energetic particle spectrometer RAPID (Figure 2a) in the Cluster payload features novel detection principles both for ions and electrons: For either species the instrument measures the vector velocity (V) and the energy (E). The ion detector heads (IIMS) are composed of a timeof-flight (TOF)/energy (E) telescope with a solid state detector (SSD) as the back element (Figure 2b). Species identification comes from the function $E T^{2}=A$ with $\mathrm{E}$ and $\mathrm{T}$ denoting the measured particle's energy and time-of-flight (equivalent to the particle velocity), $\mathrm{A}$ is the atomic mass of the particle. The energy range extends from 30 to $1500 \mathrm{keV}$. The advanced design of the telescope as a projection camera returns also information on the particle direction of incidence with 12 angular intervals over a $180^{\circ}$ field-of-view. A second detector system, the imaging electron spectrometer (IES), is dedicated to electrons between 30 to $450 \mathrm{keV}$. The IES head uses the optical principle of a pinhole camera with 9 angular intervals over $180^{\circ}$. Together with a sectored spin plane of the spacecraft, both systems cover the unit sphere in velocity space in a contiguous manner (Figures 2c and $2 \mathrm{~d}$ ). More detailed information about the RAPID 


\section{RAPID/CLUSTER II}

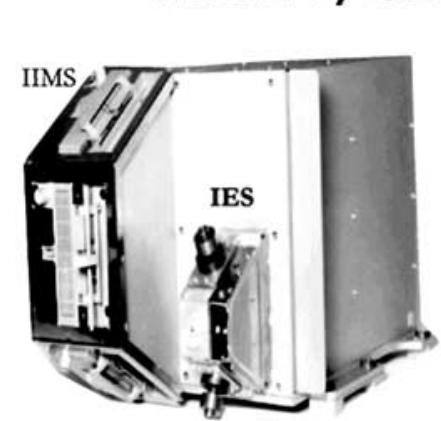

A.)

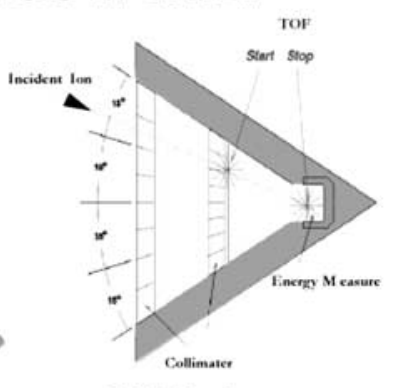

I IMS Iorgensor B.)

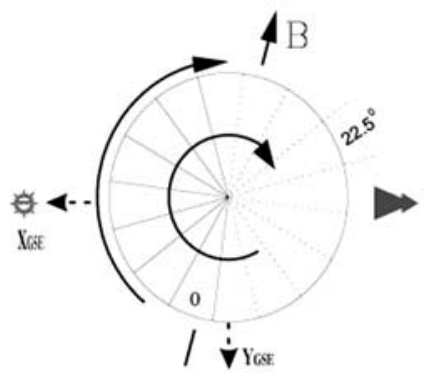

C.)

Figure 2. The RAPID spectrometer for ions and electrons A) Photograph of RAPID instrument with ion sensor IIMS on the left side and the electron sensor IES on the right side. B) The cross section of one of the three IIMS Detectors which includes the time-of-flight (T)/Energy (E) measurement system. The open angle for each detector is $60^{\circ}$, and is further divided into four $15^{\circ}$ ranges. The total of three IIMS detectors can measure the full $180^{\circ}$ polar direction with 12 angle elements. C) 16 sectors in the satellite spin plane together with the polar angular resolution allows the imaging of flux distributions over the complete unit sphere $(4 \pi)$ in phase space in a total of 192 pixels (D).

instrument is given by Wilken et al. [1998, 2001]. It should be mentioned here that the instrument is not sensitive to the ionic charge state of nuclear particles; the mass resolution is marginal for separating out carbon, nitrogen and oxygen. For the purpose of this paper the CNO group is referred to as 'oxygen' because this species is considered the major constituent. The proton data from the energy channel 27$36 \mathrm{keV}$ which is determined by a valid digital time-of-flight (TOF) signal (Direct event) has been used for the sounding purpose. The integral fluxes used in this are obtained from the following lower energy bands; for hydrogen from 27 to $70 \mathrm{keV}$, for helium from 70 to $140 \mathrm{keV}$; oxygen from 100 to $300 \mathrm{keV}$, and for electrons from 30 to $46 \mathrm{keV}$.

\section{Observation}

[6] On 14 January 2001 the geomagnetic activity was rather quiet as indicated by geomagnetic indices $D_{s t}=-1 \mathrm{nT}$ and $K_{p}=1$. The Cluster spacecraft were travelling outbound in the high-latitude region of the northern hemisphere (see Figure 3). Figure 4 gives an overview of Cluster measurements between 0800 and 1800 UT on 14 January 2001. Starting in the northern lobe, the Cluster spacecraft entered into the high-latitude boundary/cusp region at around 1040 UT and further crossed the magnetopause into the magnetosheath at about 1510 UT. The top panel in Figure 4 shows energy-integrated ion and electron fluxes versus time profiles. The second panel shows plasma velocity, Vx. The third panel shows the plasma density variation. The last four panels show magnetic field obtained by the Cluster FGM instrument [Balogh et al., 1997]. The plasma density and velocity data are from the Cluster Ion Spectrometer (CIS) experiment [Reme et al., 1997].

[7] A representation of energetic protons detected by the RAPID instrument in three dimensions is displayed in Figure 5. The angle-angle plot displays the data taken in the spacecraft frame with the Sun in sector 13. At this point, it should be noted that charged particles are guided and ordered by the magnetic field in space and as a result, the angular distributions can reveal the direction of the magnetic field vector.

[8] Figure 5 displays the entire pass through the trapping region from polar cap/lobe field lines at 1041 UT to the exit into the magnetosheath near 1511 UT. The slow evolution of the loss cones in the angle-angle distributions should be noted. The centers of these loss cones mark the entry and exit of the magnetic field vector through the unit sphere due to the special position of Cluster at the time of observation and for the display format this means that the B-vector enters the individual frame on the dusk side, which is on the left, and leaves it in the center, which is roughly towards the Earth. A trapped particle on a closed field line oscillates

\section{CLUSTER ORBIT}

\section{1/01/14 $\triangle S / C 4=$ TANGO $\triangle S_{S} C_{-}=$SALSA 12:00:00-18:00:00 $*$ SIC_3 $_{-}=$SAMBA $\square$ SIC_ $1=$ RUMBA Tetrahedron $(x 10)$}
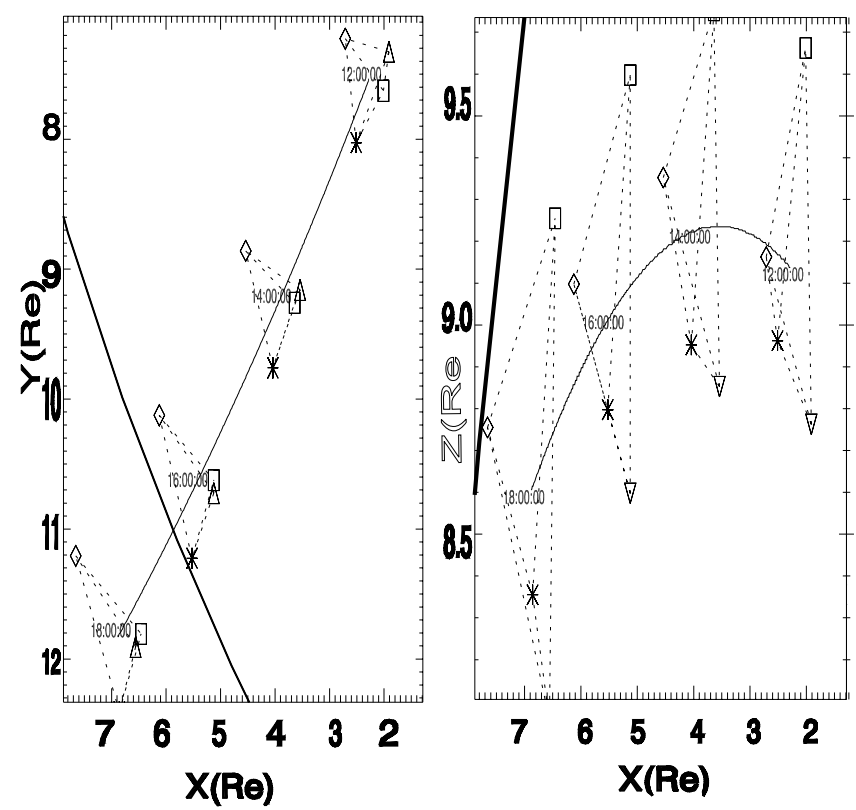

Figure 3. The Cluster trajectory on 14 January 2001. The nominal positions (unlabelled solid line) of the magnetopause have been scaled according to the model given by Fairfield [1971]. 


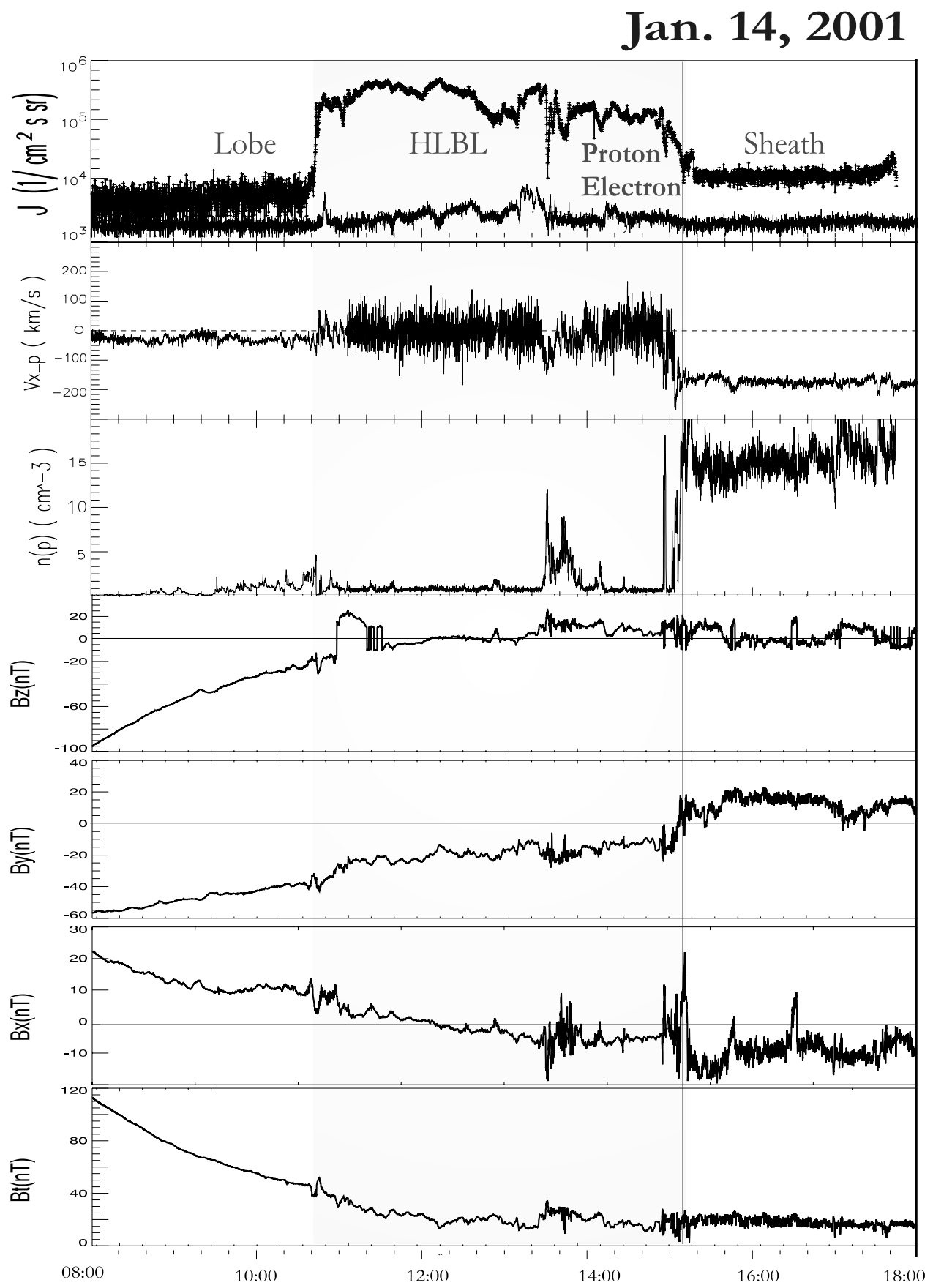

Figure 4. An overview plot of energetic particle, plasma and magnetic field obtained by Cluster (Salsa) from 0800 to $1800 \mathrm{UT}, 14$ January 2001 . In the top panel are plotted proton $(28-69 \mathrm{keV})$ and electron (30-46 keV) fluxes. The plasma velocity Vx and plasma density obtained by CIS-HIA/Cluster (energy range from $0.005-32 \mathrm{keV}$ ) are given in the second and third panels. The magnetic field components and magnitude (in $\mathrm{nT}$ ) are shown in the bottom four panels. The vertical lines mark the different regions: lobe, HLBL, and the sheath which Cluster experienced during its outbound crossing on 14 January 2001.

between north and south mirror points, at which all the particle velocity is converted to perpendicular gyration, i.e., the pitch angle is $90^{\circ}$. The further away from the maximum mirror field a particle observation is made, the smaller the width of the loss cone. This assumes that there exists a minimum loss cone at the point of minimum field strength along the field line (usually at the equator). For RAPID the pixel size is $15^{\circ} \times 22.5^{\circ}$ so that any observable loss cone must be comparable or larger than this field of view. The loss cone widths in Figure 5 increase slowly from 1131 UT. It is quite obvious that the angular width of the loss cones starts with small values at 1131 UT and grows continually until 1330 UT after which, the loss cones are mostly equal except for subtle differences over short periods of time. However, at about $1330 \mathrm{UT}$, the loss cone widths grow faster than before and reach values near $180^{\circ}$ in the vicinity 


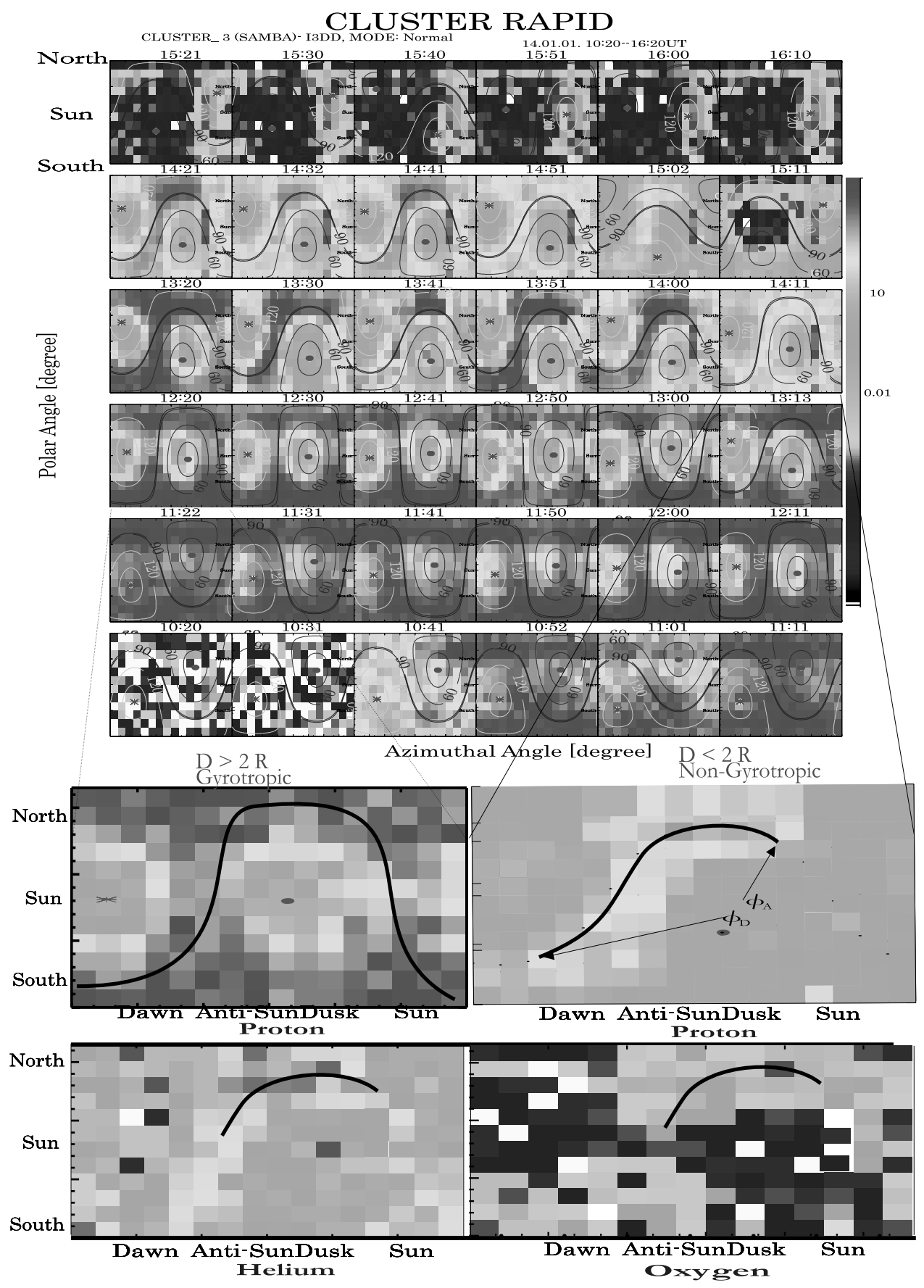

Figure 5. Three-dimensional polar versus azimuthal angle distributions of hydrogen ions for Cluster-3, Samba. $\odot$ (red) indicates the magnetic field vector $\vec{B}$ at this point into the unit-sphere (RAPID detector); * (red) marks the magnetic field vector $\vec{B}$ at this point out the sphere (RAPID detector). When the distance is larger than two gyro radii from the magnetopause, a trapped particle distribution appears gyrotropic (e.g., proton distribution at 1131 UT); when less than two gyro radii from an absorbing boundary the trapped particle distribution appears nongyrotropic (e.g., proton, helium and oxygen distribution at $1411 \mathrm{UT}$ ). See color version of this figure at back of this issue.

of the magnetopause at around 1511 UT. The actual magnetopause current layer, according to the magnetometer, is crossed at around 1509 UT.

[9] At 1041 UT, Cluster entered the trapped region at high latitudes on the duskside and left the magnetosphere at 1511 UT. The region between these boundary crossings was dominated by significant gradients in the particle intensity with many individual distributions demonstrating the azimuthal asymmetries associated with the remote sounding method described in section 1. A detailed examination of the angle-angle plots (Figure 5) shows considerable differences from time to time which may be 


\section{Magnetopause Sounding , Jan 14, 2001}

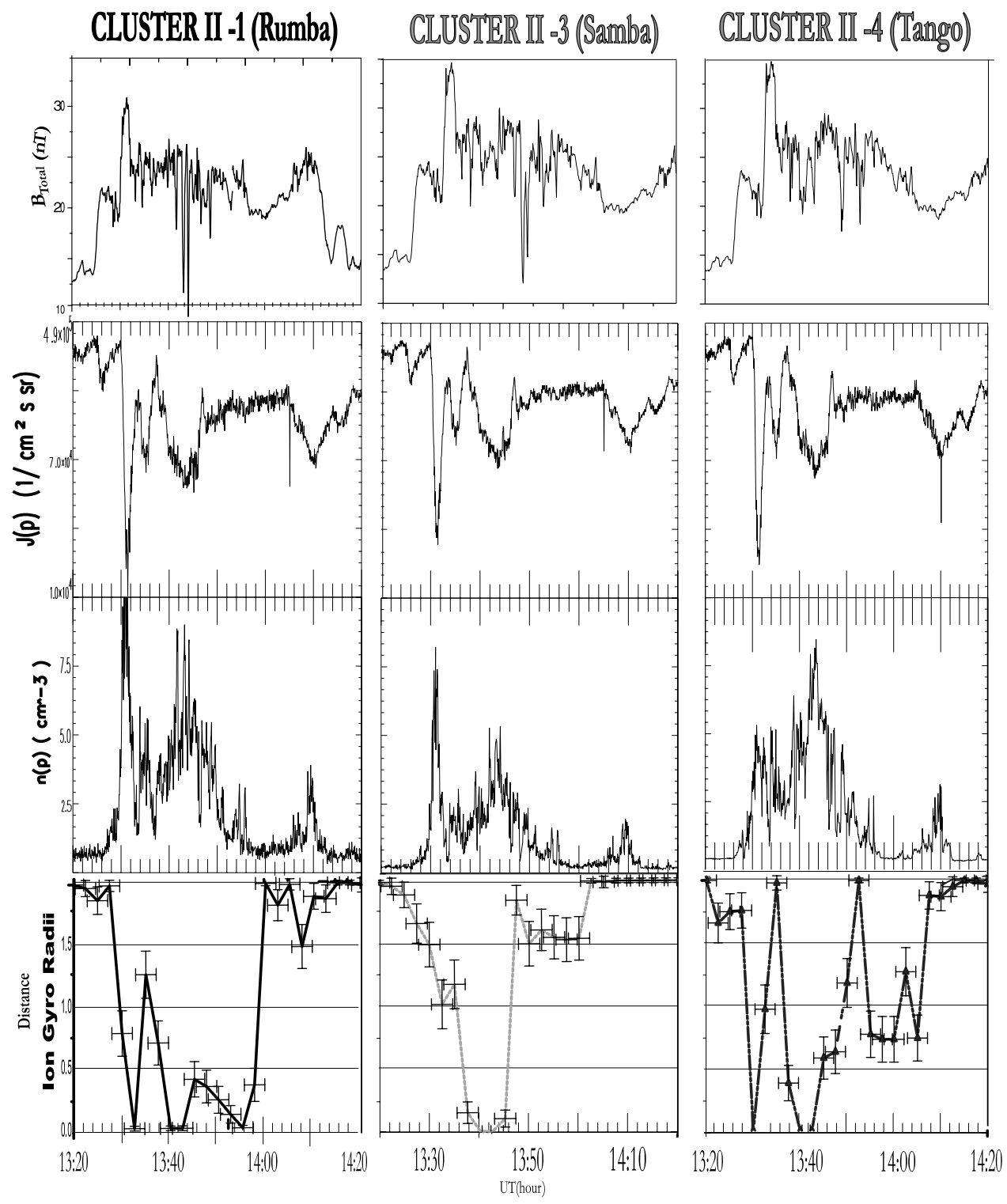

Figure 6. The energetic particle flux $(>30 \mathrm{keV})$ and the contiguous determinations of the distance between the different Cluster S/C - Rumba, Samba and Tango and the magnetopause boundary for the interval from 1320 to 1420 UT, 14 January 2001.

the result of the changes in the structure of the boundaries near the magnetopause. This is also true for different ion species, e.g., hydrogen, helium and oxygen ions. The distance between the Cluster $\mathrm{S} / \mathrm{C}$ and the magnetopause boundary for the interval from 1320 to 1420 UT, 14 January 2001, has been continuously determined. The results and their corresponding energetic proton fluxes are given in Figure 6. Figure 6 summarizes remote soundings in the interval from 1320 to 1420 UT for three Cluster satellites: Rumba, Samba and Tango. Plotted in the bottom panel of Figure 6, the distance (in proton gyro-radii) gives an overall picture of how the magnetopause boundary was coming towards, just passing, and then retreated away from the spacecraft. For comparison, the proton flux is plotted in the second panel from the top for each spacecraft. As we can see from Figure 6, the various ion intensities appear to be very similar yet the distances (in proton gyro-radii) to the magnetopause vary considerably. The distance variations between the $\mathrm{S} / \mathrm{C}$ and the magnetopause is obtained by the remote sounding technique [Oksavik et al., 2002].

[10] The distances to the magnetopause inferred by sounding ions are plotted in terms of their gyro-radii (from 0 to 2 Radii) in Figure 6. The top panel in Figure 6 shows that the total magnetic field varies from 10 to $30 \mathrm{nT}$. When we compute the real distance to the magnetopause (in $\mathrm{km}$ ), the true sounding distance $R_{i}$ must incorporate the variation of magnetic field $B_{i}$ since $R_{i}=\frac{B_{0}}{B_{i} R_{0}}$. 


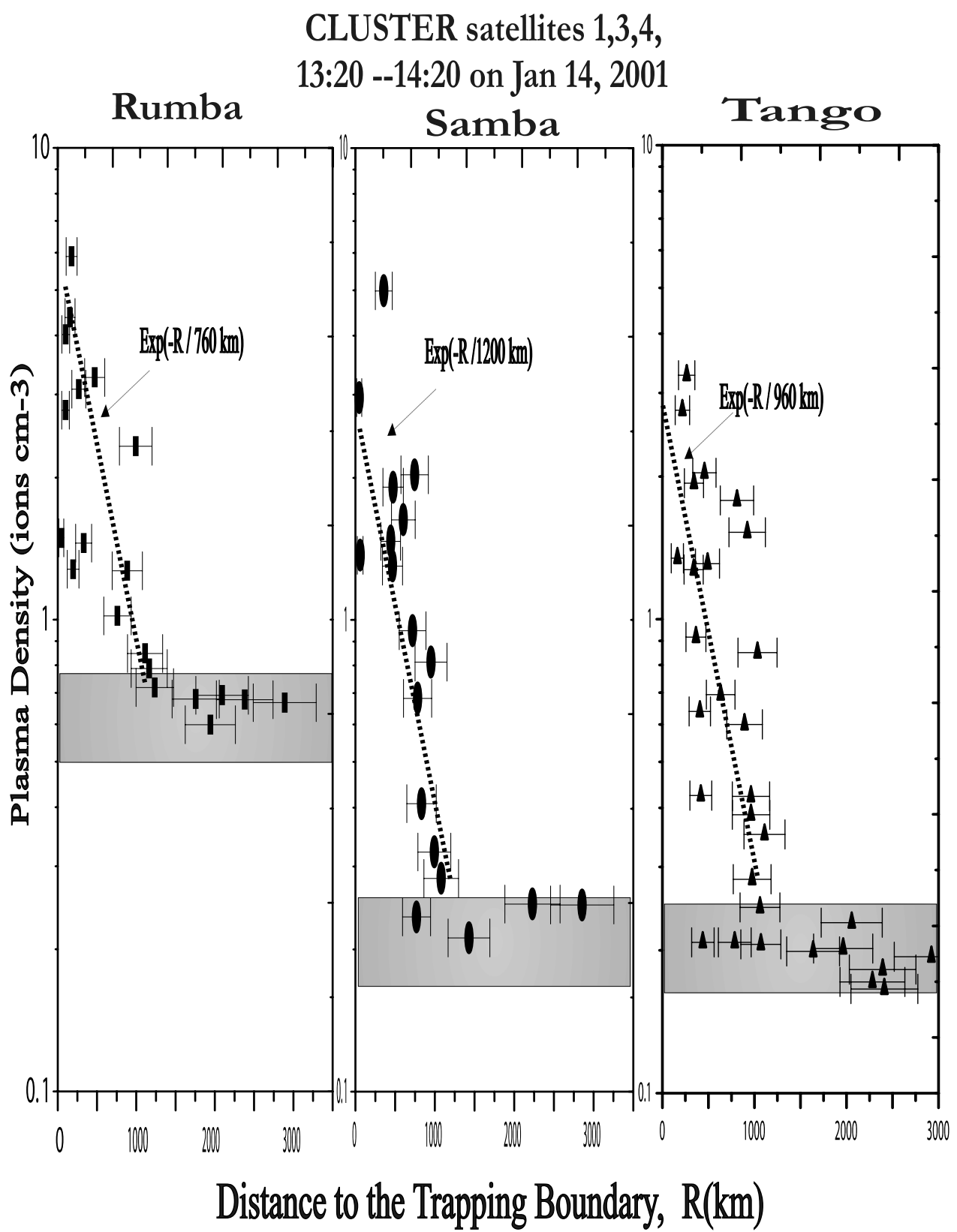

Figure 7. Reconstruction of the plasma density and the contiguous determinations of the distance between the different Cluster S/C (Rumba (square), Samba (circle) and Tango (triangle)) and the magnetopause boundary for the interval from 1320 to 1420 UT, 14 January 2001.

[11] By knowing the distance to the magnetopause, we also know the variation of the energetic particle fluxes, bulk plasma parameters, and magnetic field up to a range of $2700 \mathrm{~km}$ ( 2 gyro radii of $30 \mathrm{keV}$ proton) from the magnetosphere boundary. The density of the plasma versus the distance to the magnetopause is plotted for all available satellites in Figure 7. Here we show the appearance of solar wind plasma versus the linear distance to the magnetopause, or, depth of penetration. The horizontal (distance) error bars are the uncertainties for the simultaneous-trapping-boundary-to-Cluster distance determinations. Figure 7 shows that these data appear to be bounded by an exponential with an e-folding distance of $760 \mathrm{~km}$ (Rumba/Cluster 1), 1200 km (Samba/Cluster 3), and $960 \mathrm{~km}$ (Tango/Cluster 4). The solar wind plasma penetrates interior to the sounding boundary layer (inside the trapping boundary layer). The different baselines (plasma density) seen by the three spacecraft may imply the relative location of the satellite to the magnetopause, with Cluster/Rumba being closer to the magnetopause where the density is higher.

[12] Since the RAPID instrument is not sensitive to ionic charge state, the gyro radii of helium and oxygen ions can not be uniquely identified because the ion gyro radii is a function of charge state $\left(\rho=\frac{m v}{q B}\right)$. Nevertheless, the distance to the magnetopause can be determined uniquely from the proton measurement. As shown in Figure 8 , the fluxes of energetic particles (hydrogen, 
Jan.14, 2001

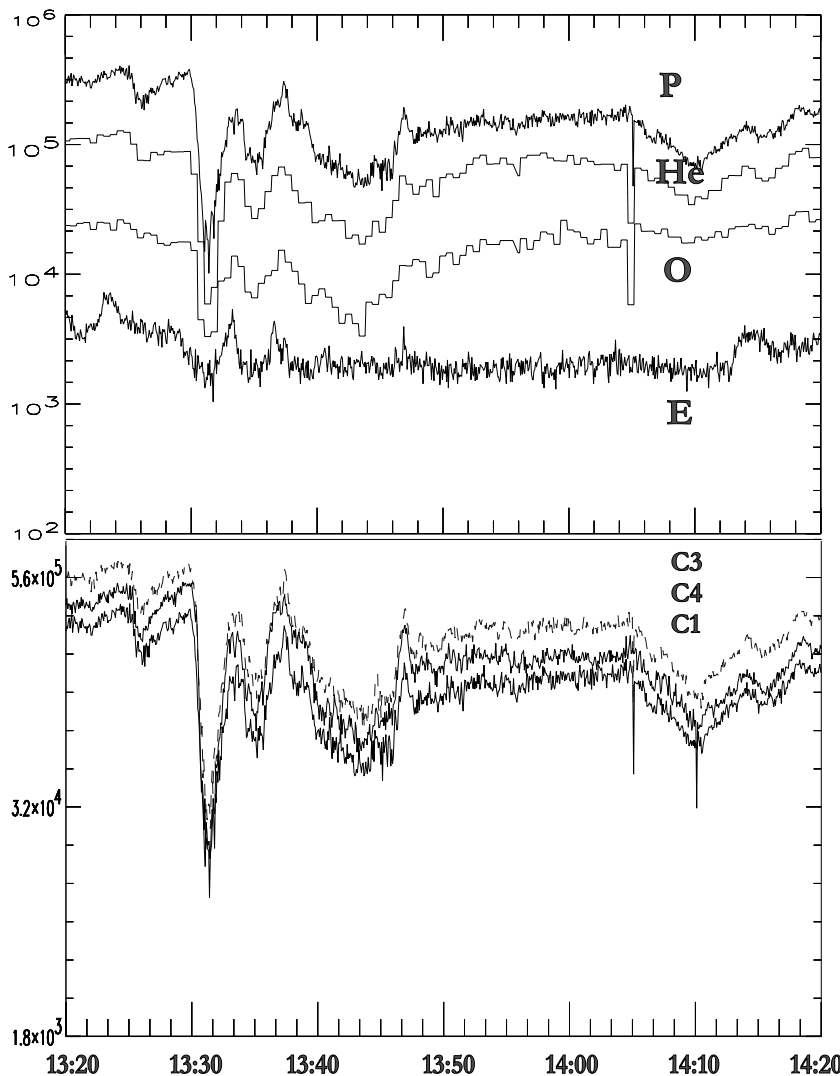

Figure 8. Upper panel: Energetic particle species flux variation from 1320 to 1420 UT, 14 January 2001 obtained by $\mathrm{S} / \mathrm{C} 1$. From the top the panels show proton, helium, oxygen, and electron flux. Bottom panel: Proton flux variation from 1320 to 1420 UT, 14 January 2001, for the different Cluster spacecraft.

helium, and oxygen ions and electrons) fluctuated in unison although the IMF was northward and geomagnetic activity was very quiet during this interval. In order to inspect the energetic flux variation for different ion species in the high-latitude boundary/cusp region in more detail, the fluxes of energetic particles from 1320 to 1420 UT are plotted as a function of the distance to the magnetopause as derived from the proton sounding (Figures 9, 10, and 11).

[13] Using this mapping, we find that the fluxes of all ion species, (hydrogen, helium and oxygen) are proportional to their computed distance from the magnetopause. The linear fits for proton, helium and oxygen flux (for all satellites, particles $\left./ \mathrm{cm}^{2} \bullet s \bullet s r\right)$ are

$$
\begin{aligned}
& \text { Flux }_{\text {proton }}=10^{5}+100 \times \text { Distance }(\text { in } \mathrm{km}), \\
& \text { Flux }_{\text {helium }}=5 \times 10^{4}+6 \times \text { Distance }(\text { in } \mathrm{km}), \\
& \text { Flux }_{\text {oxygen }}=9 \times 10^{3}+2 \times \text { Distance }(\text { in } \mathrm{km}) .
\end{aligned}
$$

[14] The correlation coefficients are $0.7,0.7$, and 0.8 , respectively. These flux gradients imply that the energetic proton, helium, and oxygen "absorbed" by the magneto- pause are 100,6 , and 2 particles $/ \mathrm{cm}^{2} s s r / \mathrm{km}$, respectively. In contrast, the energetic electrons are found to have almost no correlation with distance (the correlation coefficient is only 0.2 ). The best fit is

$$
\text { Flux }_{\text {electron }}=2 \times 10^{3}+0.1 \times \text { Distance }
$$

The very low correlation coefficient for the electrons is easily explained because the $30 \mathrm{keV}$ electron gyro radii (about $24 \mathrm{~km}$ ) is much smaller than that of the ions. The "absorbing" effect does not work for electrons at these distance scales (see Figure 12). As noted before, if the satellite is close enough to the magnetopause (i.e., the distance must be less than two ion gyro-radii), particles with some arrival directions can not complete their gyro orbits and will contact the magnetopause and be scattered [Fritz and Fahnenstiel, 1982; Zong et al., 2000]. Therefore the energetic ion flux will be decreased at those scales while the electrons (at the same scale) will not and this effect can be seen in Figure 6.

[15] The proton flux (bottom panel in the Figure 8) detected by different satellites is slightly different in an orderly manner. The highest flux was seen by C3 (Tango, the satellite with the smallest GSE Z value, see Figure 3) and the lowest flux was detected by $\mathrm{C} 1$ (Rumba, the satellite with the largest GSE Z value; see Figure 3). This indicates that the location of the magnetopause was mainly north of the Cluster satellite, and the satellite $\mathrm{C} 1$ is closer to the magnetopause than $\mathrm{C} 3$ and $\mathrm{C} 4$. This is indeed consistent with the picture obtained by the sounding and demonstrated in Figure 7.

\section{Discussion and Conclusion}

[16] Figure 12 demonstrates remote sensing of a boundary that empties gyro-orbits when the centers of those gyroorbit cross it. The notion that particle fluxes are reduced by an absorbing boundary, assumed to be the magnetopause, was used by most of the earlier studies on magnetopause sounding [e.g., Fritz and Fahnenstiel, 1982; Daly et al., 1984]. The energetic particle morphology at the magnetopause has been discussed [Paschmann, 1984; Fritz and Williams, 1984]. The "sounding" of the magnetopause by energetic particles has been further interpreted more physically as the trapping boundary or last closed field line [Fritz and Williams, 1984].

[17] At a trapping boundary (last closed field line) where the magnetic field changes configuration, gyro-orbits will be severely disturbed. Ideally, at a discontinuous uniform boundary, if any part of an orbit crosses the boundary, particles will be removed. For this boundary to be seen, there must of course be a source population on the particle-rich side as is the case in Earth's magnetosphere. In reality, the absorbing boundary is not so ideal. The temporal randomness and spatial nonuniformity of the magnetic field rotation mean many ions can indeed return from a boundary encounter. Or, one can observe those reentering after scattering from other gyro-orbits. This effect might explain the degree of scatter of points in Figure 10 relative to the linear trends of the measured gradients. Those ions populating the most external gyroorbits would be originating from only a limited range of 


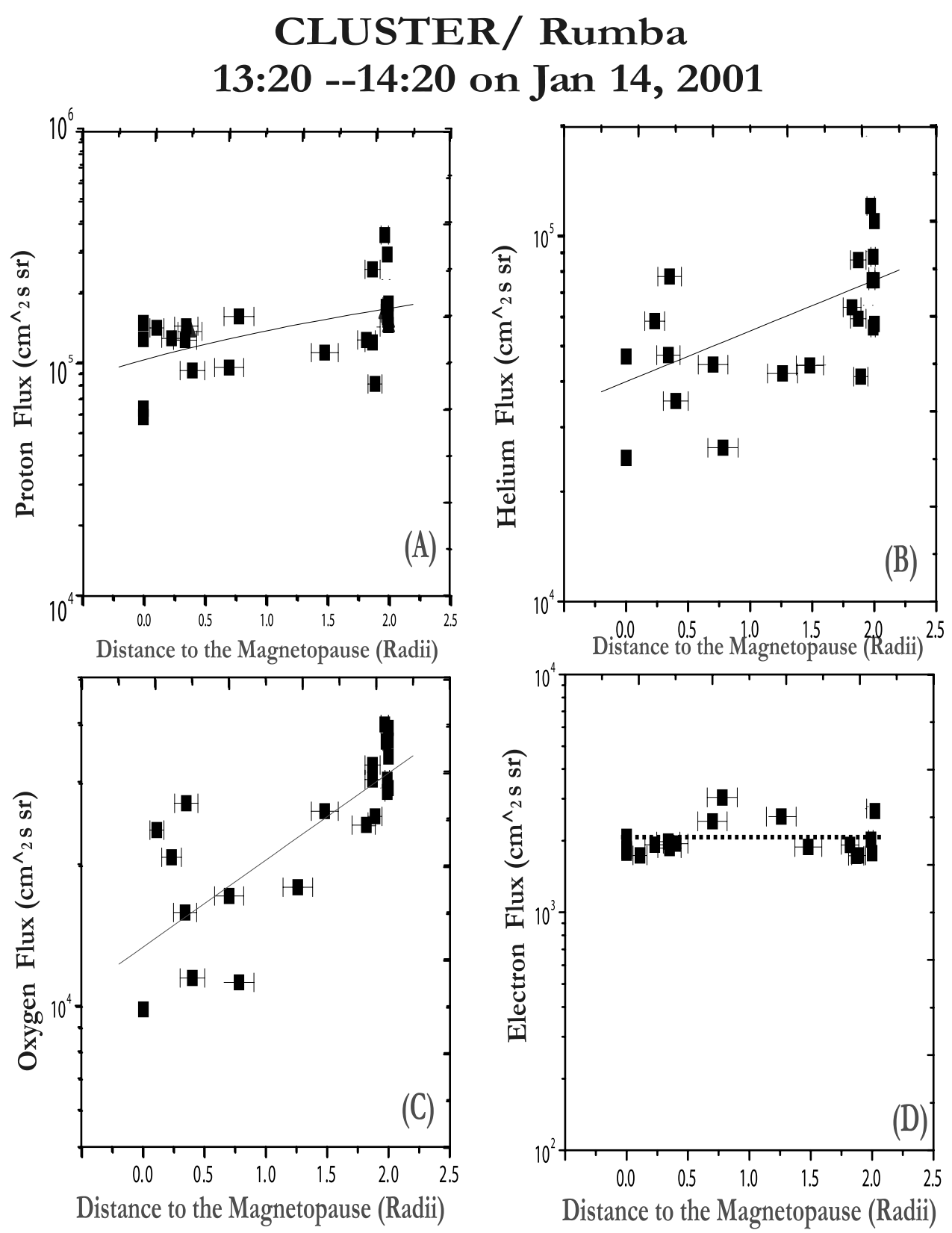

Figure 9. The fluxes of energetic particles as a function of the distance to the magnetopause (in $\rho_{H^{+}}$) which is derived by the particle sounding method. A, B, C, D for energetic particle species hydrogen, helium, oxygen, and electron, respectively.

gyrophases. Thus, when gyro-averaged, such orbits would still show a reduced density.

[18] A summary sketch of the inferred orientation and the structure of the magnetopause boundary with respect to the four Cluster spacecraft trajectories is shown in Figure 13. Energetic particles in the adjacent magnetopause boundary will depend critically on whether they are resupplied efficiently or not. Figure 13 illustrates schematically two limiting cases; the upper panel shows the scenario with inefficient resupply while the bottom panel shows the case with efficient resupply (bottom panel in Figure 13).

[19] As illustrated in Figure 13, without efficient resupply energetic particles should only be observed on the field lines which remain closed. In between the inner and outer separatrix field lines, the region will become should be depleted within a bounce period [Paschmann, 1984].

[20] The bounce period of a $30 \mathrm{keV}$ proton in a dipole field at $L=8$ is about $110 \mathrm{sec}$. This means no particles should be observed on open field lines after $110 \mathrm{sec}$ if there is no further supply. However, the particles can be supplied by, for example, longitudinal drift, if the extent of the reconnection region is not much larger than the longitudinal drift per bounce period [Zong et al., 2001]. If the reconnection region is large in longitudinal extent, again no particles should be seen on field lines in which the distance from the boundary of the reconnection region 
CLUSTER/Samba 13:20 --14:20 on Jan 14, 2001
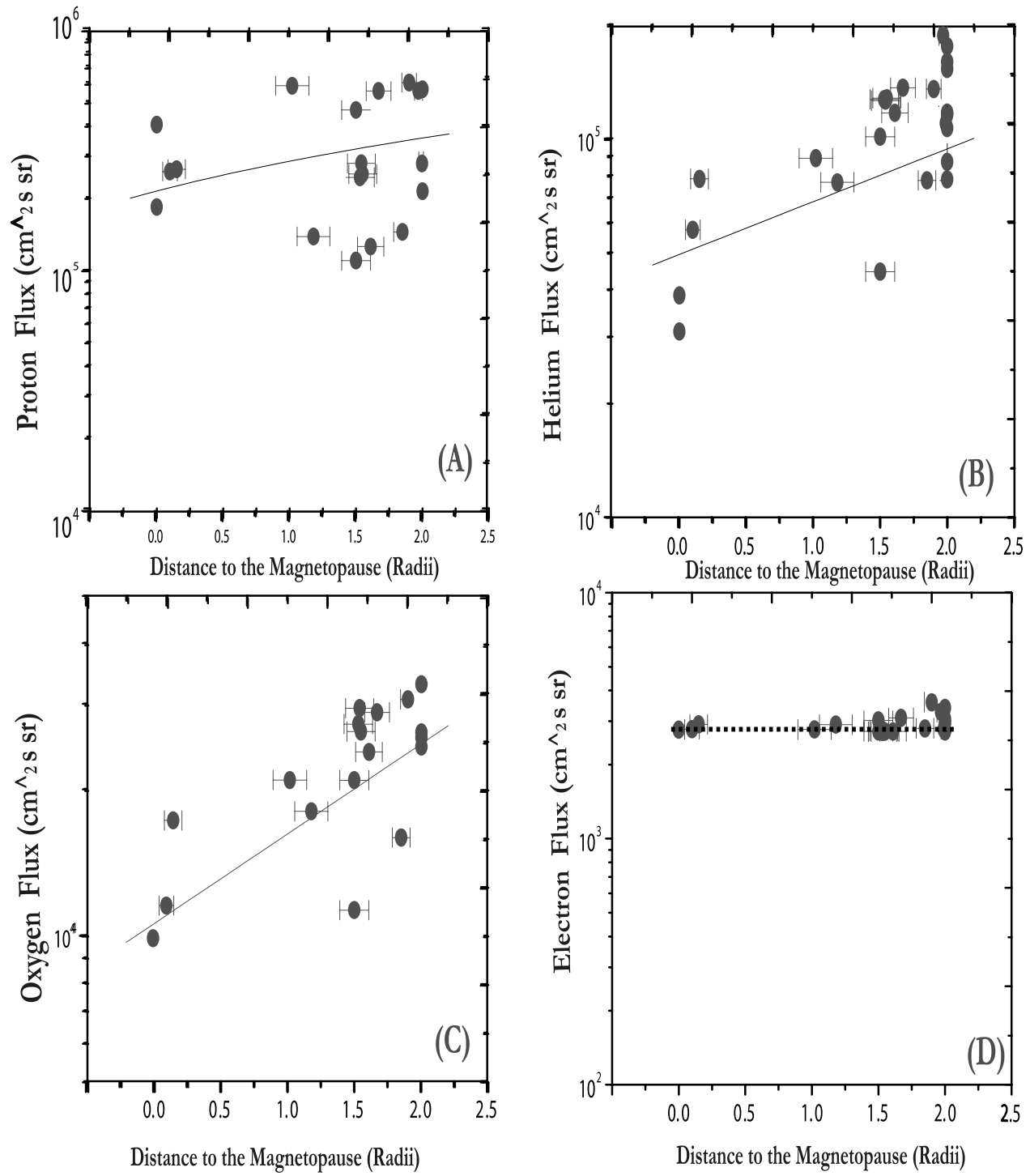

Figure 10. The same as Figure 9, but for Cluster 3 (Samba). 
CLUSTER/ Tango

13:20 --14:20 on Jan 14, 2001
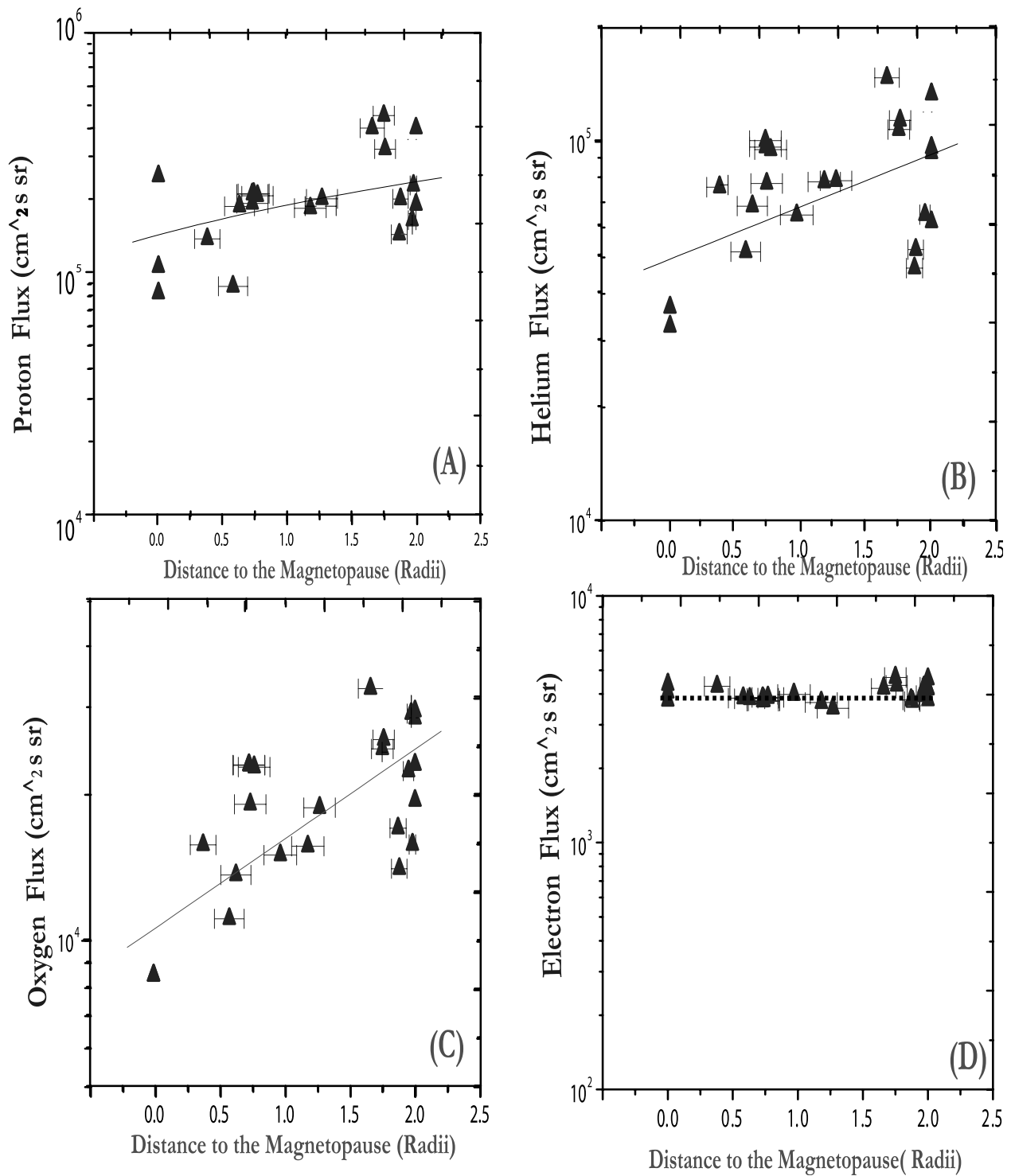

Figure 11. The same as Figure 9, but for Cluster 3 (Samba). 


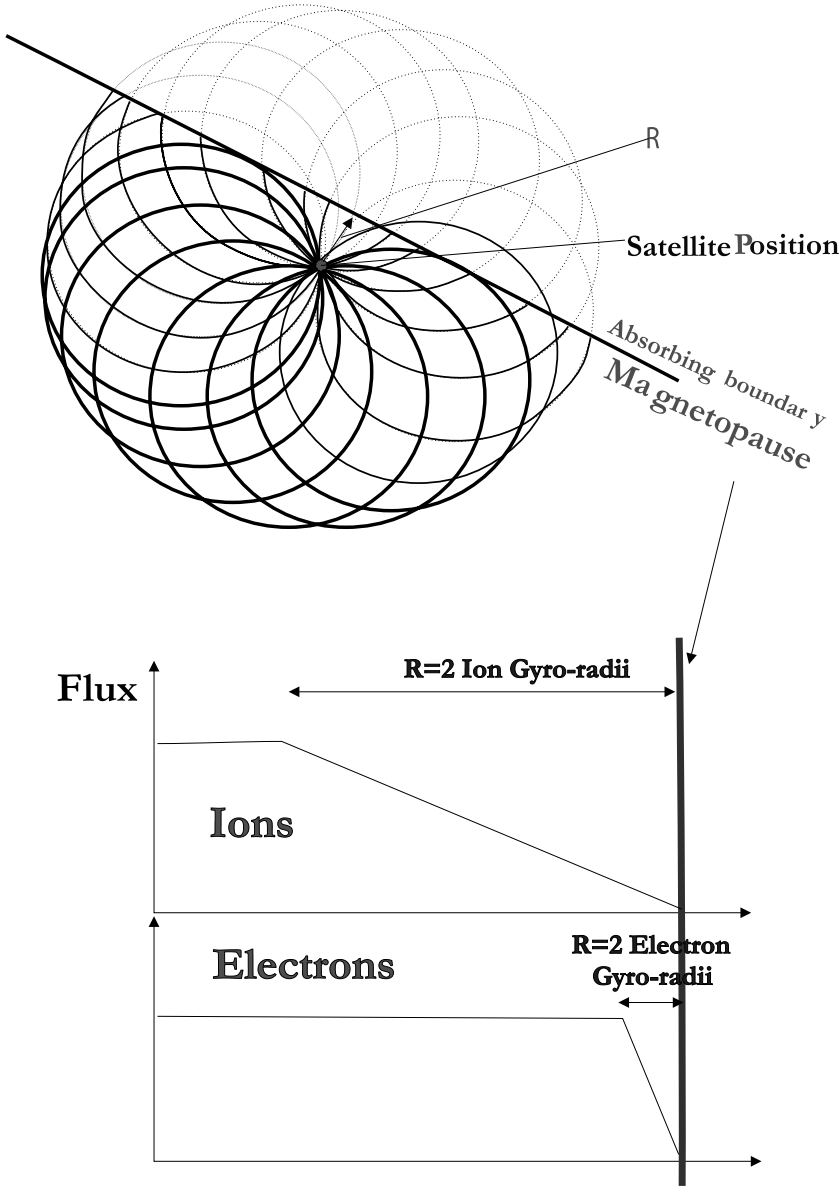

Figure 12. Snapshot of the boundary position, thick circles represent ion orbits that do not cross the absorbing boundary and therefore exhibit high intensities, while thin arcs are those parts of the trajectory connection to the boundary, and dotted arcs are nonexistent trajectories on the other side of the boundary.

is larger than the longitudinal drift path within one bounce period [Zong et al., 2001].

[21] The gyro-diameter of the sounding ion in this paper is about $2700 \mathrm{~km}$ (the electron diameter $2 \rho_{e}$ is $50 \mathrm{~km}$ ), a factor of about 2 greater than our inferred solar wind plasma penetration distance of $1500 \mathrm{~km}$. This is rather thick if we compare it with the thickness of the magnetopause found in the subsolar region. The magnetopause thickness is usually between 200 to $1000 \mathrm{~km}$ [e.g., Russell, 1995]. A systematic study of low-latitude boundary layer was carried out by Phan and Paschmann [1996] and Phan et al. [1996] using the AMPTE/IRM data. The average combined width of the magnetopause and LLBL was found to be about $1240 \mathrm{~km}$. This thickness is in agreement with the result obtained in this paper, namely the penetration distance of solar wind plasma, although our observation was made in the highlatitude region. These results confirm that solar wind plasma occupies a region interior to the energetic particle trapping region (see Figure 13). Since the plasma density started to decay at the boundary (the distance " 0 ": Figure 7), this suggests the observed topology is more consistent with the scenario proposed in the upper panel of Figure 13 assuming that the thickness between the inner and outer separatrix field lines (magnetopause boundary layer) is larger than $300 \mathrm{~km}$ (uncertainty in distance). The solar wind plasma is indeed present more than $\approx 1000 \mathrm{~km}$ from the trapping boundary.

[22] The term "inner edge of the boundary" (IEBL) is adopted from Phan and Paschmann [1996] and Phan et al. [1996], who define the IEBL as the location where the density has dropped to $5 \%$ of its magnetosheath value. This definition is quite consistent with the result shown here in Figure 7. Whether the IEBL is formed by a further penetration of solar wind plasma into the trapping regions on closed field lines via diffusion process (e.g., eddy effect [Haerendel et al., 1978]) or the appearance of magnetosheath plasma on interconnected field lines whose flux tube has not had time to become empty of its ion population, remains an open question. The last picture is consistent with reconnection [Lockwood and Smith, 1992; Onsager, 1993]. For this time period, between 1320 and 1420 UT, 14 January 2001, the geomagnetic activity was very quiet as indicated by geomagnetic indices $D_{s t}=$ $-1 \mathrm{nT}$ and $K_{p}=1$. Observations reported in this paper occurred during a period of extended small northward IMF $\mathrm{Bz}$, with generally larger By and Bx. Opgenoorth et al. [2001] have suggested a minor excursion of $\mathrm{Bz}$ toward small values between 1305 and 1312 UT which, together with a negative excursion of By, allowed for the occurrence of subsolar (Bz southern type) reconnection in the northern hemisphere prenoon region [Lockwood et al., 2000].

[23] Alternatively, if reconnection does occur at high latitude in both the north and south hemispheres [Lockwood and Smith, 1992; Onsager, 1993], reconnection will form a boundary layer with closed field lines. Reconnection in the northern hemisphere lobe generates "overdraped lobe" field lines that drape over the dayside magnetosphere, the "overdraped" lobe field lines generated by the reconnection in northern hemisphere lobe [Crooker, 1992] are later reclosed by reconnection in the southern lobe. The occurrence of reconnection at highlatitudes in both hemispheres has been suggested as a source of high-altitude LLBL plasma on the closed field lines [Song and Russell, 1992]. The newly added magnetospheric flux tube has lower density than the magnetosheath flux tubes, but it has higher density than other magnetospheric flux tubes. Then, the solar wind plasma we see within the trapping boundary $\approx 1000 \mathrm{~km}$ can be explained as the newly formed LLBL: solar wind plasma remains on closed flux tubes that have not had time to empty.

[24] Our results clearly illustrate how energetic ions can be used to remotely sense the local magnetopause in three dimensions, when a spacecraft is within two ion gyro-radii from a scattering boundary like the magnetopause. Knowing the local magnetic field, our method returns a distance and tilt angle of the magnetopause for each spacecraft, each ion species ( $\mathrm{H}, \mathrm{He}, \mathrm{CNO})$, and each ion energy. Making an assumption about the shape of the magnetopause, it is possible to remotely sense the motion and direction of the three-dimensional local magnetopause surface within two ion gyro-radii from the Cluster spacecraft, limited by the 
Energetic particle without resupply

IEBL

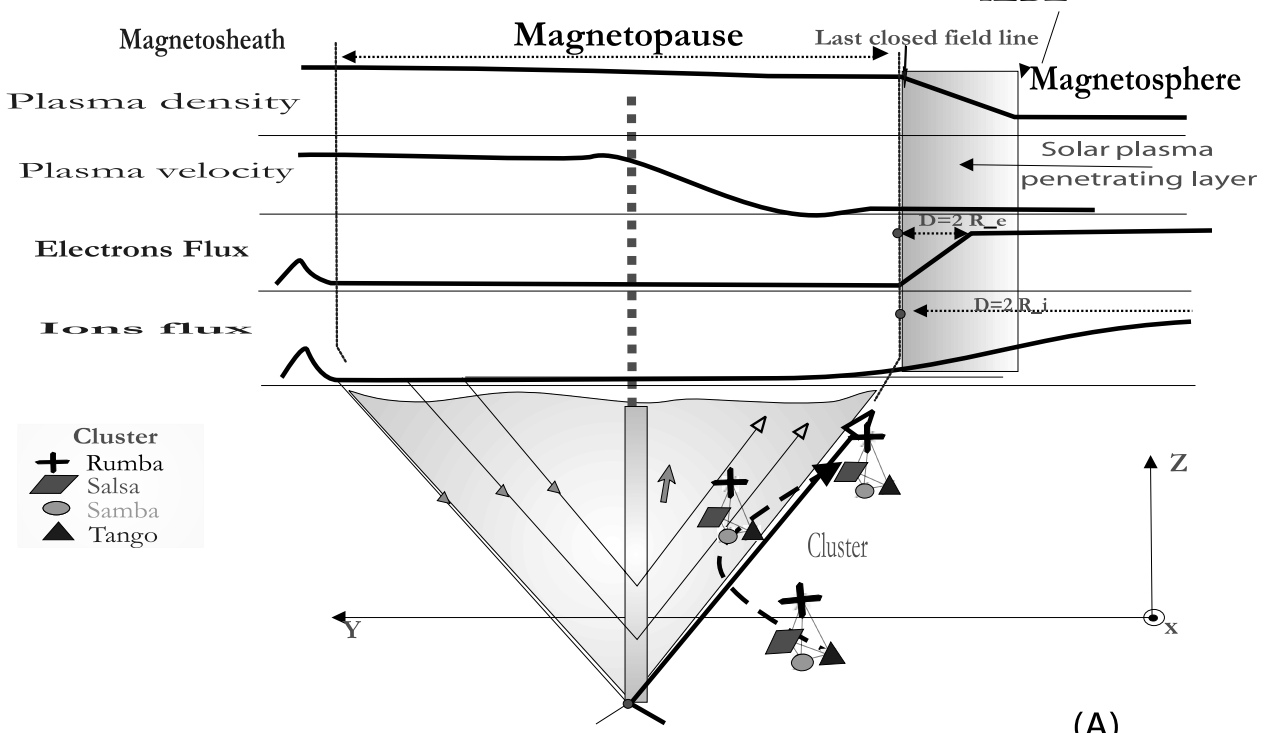

(A)

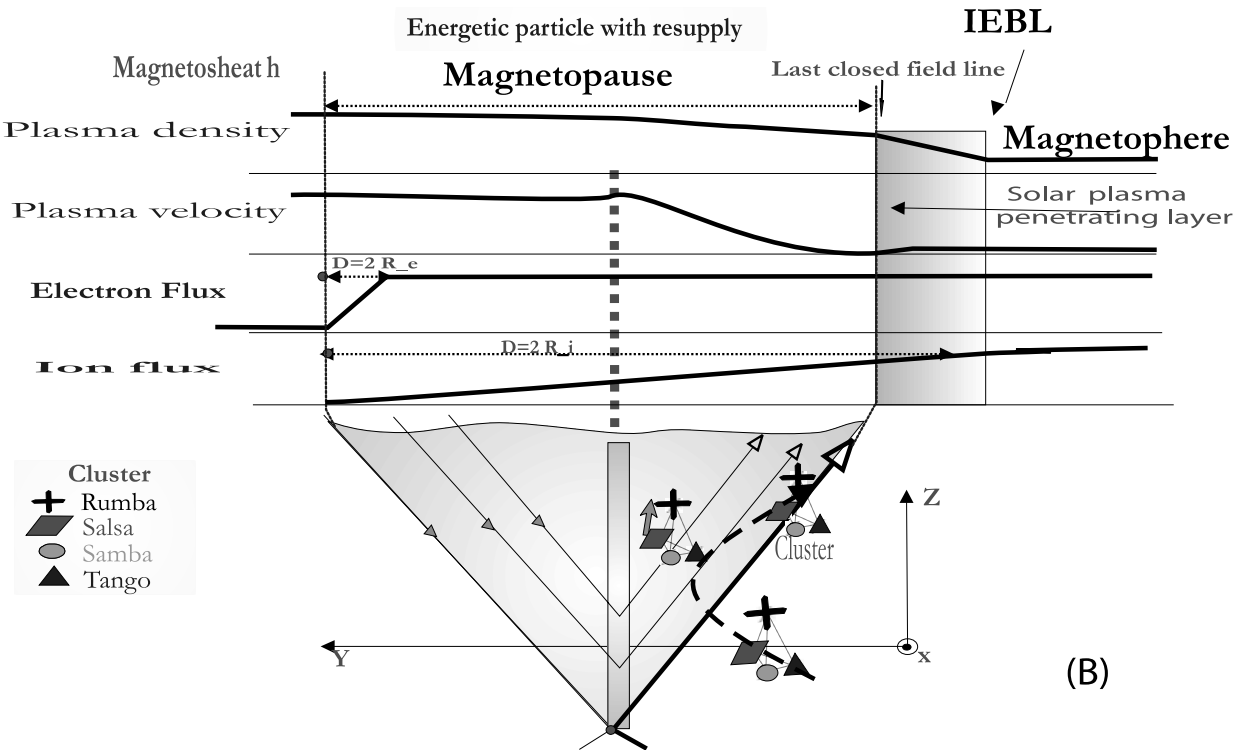

Figure 13. Schematic diagram showing the energetic particle intensity profiles in the vicinity of the high-latitude boundary layer which should be observed without (A) and with (B) efficient resupply. Signatures in plasma density and plasma speed are also shown. The last closed field line or trapping boundary layer is indicated. IEBL represent the inner edge of the boundary.

accumulation time (128 seconds) of the RAPID data set which we use for sounding in this paper.

\section{Summary}

[25] To summarize, the energetic particle measurements made in the vicinity of the high-latitude magnetopause boundary layer have been used to infer the structure and topology of that region in a remarkable manner: We conclude that: (1) The distance profiles obtained by the remote sounding method are closely related to the flux variation of the energetic particles. Variability about the linear fit between flux and distance is likely due to uncertainty in the distance measurement. All the ion fluxes are proportional to the distance from the magnetopause, with the correlation coefficient between 0.7 and 0.8 . The proton flux gradient caused by energetic protons being absorbed at the magnetopause is 100 particles $/ \mathrm{cm}^{2} s s r / \mathrm{km}$. In contrast, the plasma density is found to be inversely proportional to distance from the magnetopause. The density gradient of the solar wind plasma penetrating into the magnetosphere is found to be bounded by an exponential with an e-folding distance of $1000 \mathrm{~km}$.

[26] (2) Solar wind plasma is indeed observed interior to the trapping boundary layer inside the magnetosphere up to a depth of $1000 \mathrm{~km}$ in the high-latitude boundary 
region. This Solar wind plasma can be explained as either: (a) the newly formed LLBL with closed magnetic field lines whose flux tubes have not had time to empty; or, (b) further penetration of solar wind plasma into the trapping regions on closed field lines by a diffusive process.

[27] Acknowledgments. We are grateful to the Cluster RAPID team We would also like to thank the other Cluster teams for providing summary parameters. Financial support has been provided to KO by the Norwegian Research Council and AFOSR Task 2311AS and by NASA grants NAG56926 and NAG5-10108 at Boston University.

[28] Lou-Chuang Lee thanks the two reviewers for their assistance in evaluating this paper.

\section{References}

Balogh, A., et al. (1997), The Cluster magnetic field investigation, Space Sci. Rev., 79, 65-91.

Crooker, N. U. (1992), Reverse convection, J. Geophys. Res., 97, 19,36319,372 .

Daly, P. (1982), Remote sensing of energetic particle boundaries, Geophys. Res. Lett., 9, 1329-1332.

Daly, P., T. R. Sanderson, and K. P. Wenzel (1984), Survey of energetic $(\mathrm{E} \approx 35 \mathrm{kev})$ ion anisotropies in the deep geomagnetic tail, J. Geophys. Res., 89, 10,733-10,739.

Fairfield, D. H. (1971), Average and unusual locations of the Earth's magnetopause and bow shock, J. Geophys. Res., 76, 6700-6716.

Fritz, T. A., and S. C. Fahnenstiel (1982), High temporal resolution energetic particle sounding at the magnetopause on November 8,1977 , using ISEE 2, J. Geophys. Res., 87, 2125-2131.

Fritz, T. A., and D. J. Williams (1984), Energetic particle sounding at the magnetopause and implications for magnetic reconnection, in Proceedings of the Conference on the Achievements of the IMS, Eur. Space Agency Spec. Publ., ESA-SP 217, 131-135.

Haerendel, G., G. Paschmann, N. Sckopke, H. Rosenbauer, and P. C. Hedgecock (1978), The frontside boundary layer of the magnetosphere and the problem of reconnection, J. Geophys. Res., 83, 3195-3216.

Kaufmann, R. L., and A. Konradi (1973), Speed and thickness of the magnetopause, J. Geophys. Res., 78, 6549-6567.

Konradi, A., and R. L. Kaufmann (1965), Evidence for rapid motion of the outer boundary of the magnetosphere, J. Geophys. Res., 70, 1627-1637.

Lockwood, M., and M. F. Smith (1992), The variation of reconnection rate at the dayside magnetopause and cusp ion precipitation, J. Geophys. Res. 97, $14,841-14,847$

Lockwood, M., I. W. McCrea, S. E. Milan, J. Moen, J. C. Cerisier, and A. Thorolfsson (2000), Plasma structure within poleward-moving cusp/ cleft auroral transients: EISCAT Svalbard radar observations and an explanation in terms of large local time extent of events, Ann. Geophys., 18, 1027-1041

Lockwood, M., et al. (2001), Coordinated Cluster and ground-based instrument observations of transient changes in the magnetopause boundary layer during an interval of predominantly northward IMF: Relation to reconnection pulses and FTE signatures, Ann. Geophys., 19, 1613-1640.

Moen, J., et al. (2001), Cluster boundary-layer measurements and optical observations at magnetically conjugate sites, Ann. Geophys., 19, $1655-$ 1668.

Oksavik, K., T. A. Fritz, Q.-G. Zong, F. Soraas, and B. Wilken (2002), Three-dimensional energetic ion sounding of the magnetopause using
Cluster/RAPID, Geophys. Res. Lett., 29(10), 1347, doi:10.1029/ 2001GL014265.

Onsager, T. G. (1993), Model of magnetosheath plasma in the magnetosphere: Cusp and mantle particles at low altitudes, Geophys. Res. Lett., $20,479-482$

Opgenoorth, H. J., et al. (2001), Coordinated ground-based, low altitude satellite and Cluster observations on global and local scales during a transient post-noon sector excursion of the magnetospheric cusp, Ann. Geophys., 19, 1367-1398.

Paschmann, G. (1984), Plasma and particle observations at the magnetopause: Implications for reconnection, in Magnetic Reconnection in Space and Laboratory Plasmas, Geophys. Monogr. Ser., vol. 30, edited by E. W. Hones Jr., pp. 114-123, AGU, Washington, D. C.

Phan, T. D., and G. Paschmann (1996), Low-latitude dayside magnetopause and boundary layer for high magnetic sheath: 1 . Structure and motion, J. Geophys. Res., 101, 7801-7815.

Phan, T. D., G. Paschmann, and B. U. Ö. Sonnerup (1996), Low-latitude dayside magnetopause and boundary layer for high magnetic sheath: 2 . Occurrence of magnetic reconnection, J. Geophys. Res., 101, 78177828.

Reme, H., et al. (1997), The Cluster Ion Spectrometry (CIS) experiment, Space Sci. Rev., 79, 303-350.

Russell, C. T. (1995), The structure of the magnetopause, in Physics of the Magnetopause, Geophys. Monogr. Ser, vol. 90, edited by P. Song, B. U. O. Sonnerup, and M. F. Thomsen, pp. 81-98, AGU, Washington, D. C. Song, P., and C. T. Russell (1992), Model of the formation of the lowlatitude boundary layer for strongly northward interplanetary magnetic field, J. Geophys. Res., 97, 1411-1420.

Wilken, B., Q.-G. Zong, T. Doke, T. Mukai, T. Yamamoto, G. D. Reeves, K. Maezawa, S. Kokubun, and S. Ullaland (1998), Substorm activity on January 11, 1994: Geotail observations in the distant tail during the leading phase of a corotating interaction region, J. Geophys. Res., 103, $17,671-17,689$.

Wilken, B., et al. (2001), First results from the rapid imaging energetic particle spectrometer on board Cluster, Ann. Geophys., 19, 1355-1366.

Williams, D. J. (1979), Magnetopause characteristics inferred from threedimensional energetic particle distributions, J. Geophys. Res., 84, 101104.

Williams, D. J. (1980), Magnetopause characteristics at $0840-1040$ hours local time, J. Geophys. Res., 85, 3387-3395.

Zong, Q.-G., B. Wilken, S. Y. Fu, and Z. Y. Pu (2000), Energetic oxygen ions sounding the magnetopause, in Multiscale/Multipoint Plasma Measurements, Eur. Space Agency Spec. Publ., ESA-SP 449, 379-385.

Zong, Q.-G., B. Wilken, S.-Y. Fu, T. A. Fritz, Z.-Y. Pu, N. Hasebe, and D. J. Williams (2001), Ring current oxygen ions in the magnetosheath caused by magnetic storm, J. Geophys. Res., 106, 25,541-25,556.

Zong, Q.-G., T. A. Fritz, B. Wilken, and P. W. Daly (2002), Energetic ions in the high latitute boundary layer of the magnetosphere: RAPID/Cluster observation, in Earth's Low-Latitude Boundary Layer, Geophys. Monogr. Ser, vol. 133, edited by P. T. Newell and T. G. Onsager, pp. 101-110, AGU, Washington, D. C.

P. W. Daly and A. Korth, Max-Planck-Institut für Aeronomie, D-37191, Katlenburg-Lindau, Germany. (korth@limpi.mpg.de; daly@linmpi.mpg.de) T. A. Fritz, H. Spence, and Q.-G. Zong, Center for Space Physics, Boston University, 725 Commonwealth Avenue, Boston, MA 02215, USA. (fritz@bu.edu; spence@bu.edu; zong@bu.edu)

K. Oksavik, Department of Physics, University of Oslo, Oslo, Norway. Z.-Y. Pu, Department of Geophysics, Peking University, Beijing 100871 , China. (zypu@pku.edu.cn) 


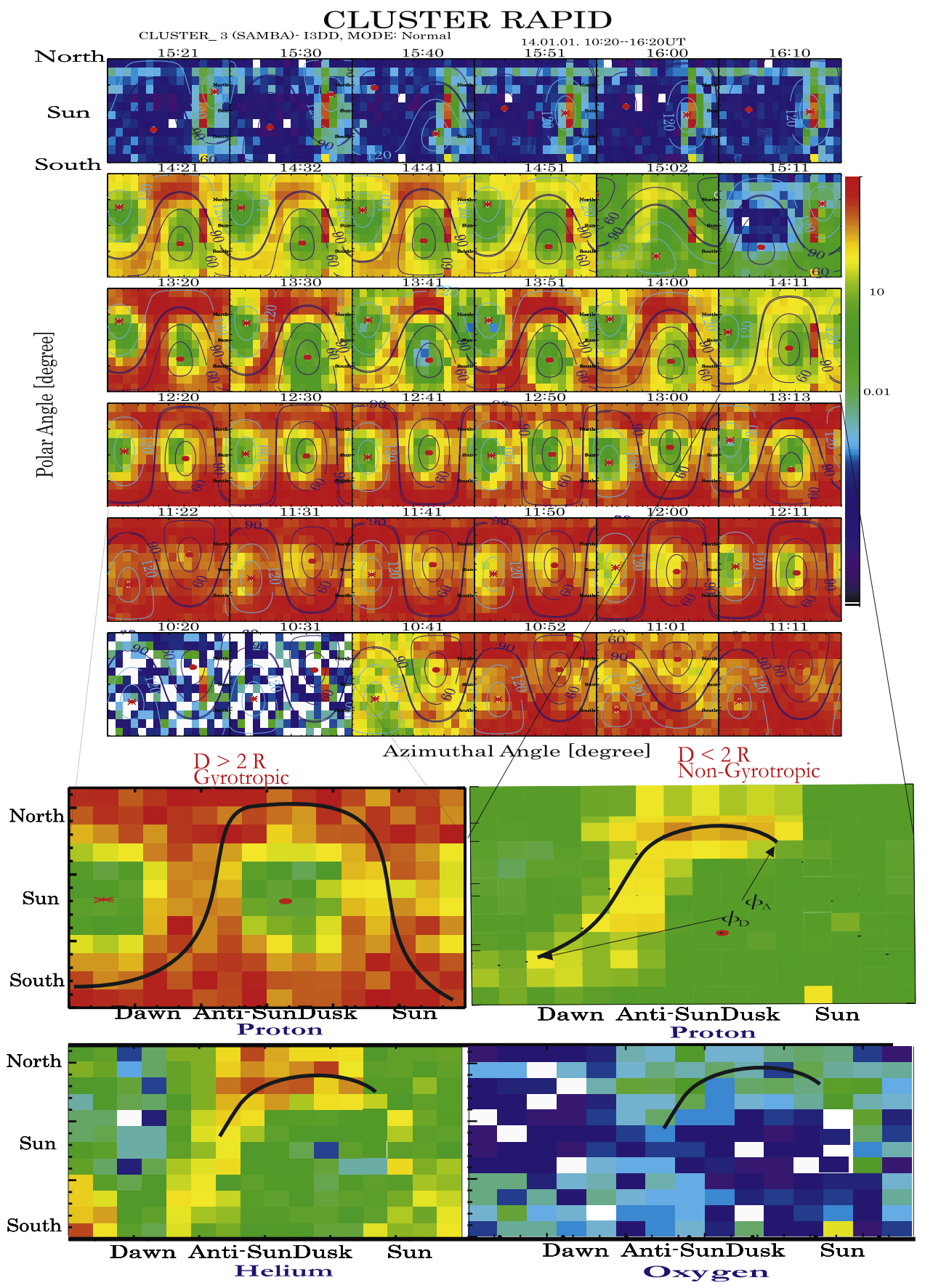

Figure 5. Three-dimensional polar versus azimuthal angle distributions of hydrogen ions for Cluster-3, Samba. $\odot$ (red) indicates the magnetic field vector $\vec{B}$ at this point into the unit-sphere (RAPID detector); * (red) marks the magnetic field vector $\vec{B}$ at this point out the sphere (RAPID detector). When the distance is larger than two gyro radii from the magnetopause, a trapped particle distribution appears gyrotropic (e.g., proton distribution at $1131 \mathrm{UT}$ ); when less than two gyro radii from an absorbing boundary the trapped particle distribution appears nongyrotropic (e.g., proton, helium and oxygen distribution at $1411 \mathrm{UT}$ ). 